\title{
Efeito de indutores no controle de míldio em Vitis labrusca
}

\author{
Regina Ceres Torres da Rosa ${ }^{1}$, Rildo Sartori Barbosa Coelho ${ }^{1}$, Selma Cavalcante Cruz de Holanda Tavares ${ }^{2} \&$ \\ Vanildo Alberto Leal Bezerra Cavalcanti ${ }^{1}$.
}

${ }^{1}$ Laboratório de Fitopatologia, Empresa Pernambucana de Pesquisa Agropecuária - IPA, Bongi, 50761-000, Recife-PE, e-mail: reginactrosa@ gmail.com. ㄹmbrapa Solos/UEP-Recife, Boa Viagem, 51020-240, Recife-PE. CNPq/ FACEPE/ PROMATA.

Data de chegada: 16/09/2005. Aceito para publicação em: 22/06/2006.

\section{RESUMO}

Rosa, R.C.T., Coelho, R.S.B., Tavares, S.C.C. de H. \& Cavalcanti, V.A.L.B. Efeito de indutores no controle de míldio em Vitis labrusca. Summa Phytopathologica, v.33, n.1, p.68-73, 2007.

A cultura da uva, Vitis labrusca, é de grande importância econômica para a região do vale do Siriji - PE. No entanto, nos últimos anos, o míldio da videira vem pondo em risco essa atividade, fazendo com que os produtores invistam bastante recurso com o uso de fungicida visando o seu controle. Visando substituir o uso de agrotóxico foi avaliado nos municípios de São Vicente Férrer - PE (área 1), Natuba - PB (área 2) e Macaparana - PE (área 3) o efeito da indução de resistência promovida por ácido DL- â aminobutírico (BABA), Agro-Mos (AM) + Crop-set (Cs), Codavit $(\mathrm{Cd})$ e Ecolife (Ec) em videiras da variedade 'Isabel'. Os resultados obtidos em relação à severidade do míldio demonstraram que o indutor $\mathrm{AM}+\mathrm{Cs}$ destacou-se dos demais tratamentos, não diferindo estatisticamente dos indutores $\mathrm{AM}+\mathrm{Cs}$ e $\mathrm{Cd}$, na área 2, e de $\mathrm{AM}+\mathrm{Cs}$ e Cd, e Ec na área 3. Quanto a eficiência de controle o $\mathrm{AM}+\mathrm{Cs}$ foi capaz de reduzir a severidade da doença em $37,46 \%, 35,97 \%$ e $18,57 \%$ nas áreas 1, 2 e 3, respectivamente. Nas áreas 1,2 e 3 a severidade da doença desenvolveu-se obedecendo as seguintes equações, gompertz $\mathrm{Y}=$

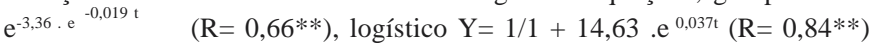
e gompertz $\mathrm{Y}=\mathrm{e}^{-4,13 \cdot \mathrm{e}-0,034 \mathrm{t}} \quad(\mathrm{R}=0,62 * *)$, respectivamente. A severidade do míldio da videira em função do tempo na área 1 , na maioria dos tratamentos, obedeceu ao modelo de gompertz, exceto o tratamento com BABA cujo modelo que se ajustou foi o logístico. Na área 2, o aumento da severidade da doença nos tratamentos estudados não apresentou predominância de um modelo. O modelo monomolecular apareceu com uma freqüência maior do que os demais, nos tratamentos da área 3.

Palavras-Chave Adicionais: Plasmopara viticola, videira, indução de resistência.

\begin{abstract}
Rosa, R.C.T., Coelho, R.S.B., Tavares, S.C.C. de H. \& Cavalcanti, V.A.L.B. Effect of elicitors in the control of downy mildew in Vitis labrusca. Summa Phytopathologica, v.33, n.1, p.68-73, 2007.
\end{abstract}

The culture of the grape, Vitis labrusca, is of great economic importance for the region of the valley of the Siriji - PE. However, in recent years, the mildew of the grapevine comes putting in rich this activity, making with that the producers sufficiently invest to resource with the fungicide use aiming at its control. Aiming at to substitute the agrotóxico use it was evaluated in the cities of Are Vicente Férrer - PE (area 1), Natuba - PB (area 2) and Macaparana - PE (area 3) the effect of the induction of resistance promoted for DL- $\beta$ - aminobutyric acid (BABA), Agro-Mos (AM) + Cropset $(\mathrm{Cs})$, Codavit $(\mathrm{Cd})$ and Ecolife $(\mathrm{Ec})$ was evaluated on grape variety 'Isabel'. Results have showed that AM + Cs was superior to the others treatments in reducing disease severity. In relation to efficiency control the $\mathrm{AM}+\mathrm{Cs}$ treatment reduced disease severity in $37,46 \%, 35,97 \%$ and $18,57 \%$ in the area 1,2 and 3 , respectively. The disease severity in the areas 1,2 , and 3 , fitted the $\mathrm{Y}=\mathrm{e}^{-3,36 \cdot \mathrm{e}-0,019 \mathrm{t}}\left(\mathrm{R}=0,66^{* *}\right), \mathrm{Y}=1 / 1+14,63 \cdot \mathrm{e}^{-0,037 \mathrm{t}}\left(\mathrm{R}=0,84^{* *}\right)$ and $\mathrm{Y}=$

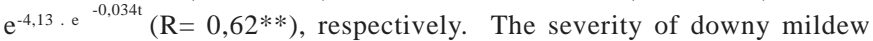
in function of the time in the area 1, fit the gompertz model, except in the BABA treatment that was described by the equation logstic. In the area 2, a predominant model did not fit data from all treatments. The monomolecular model was described more frequently than others, in the treatments of area 3.
O míldio da videira, causado por Plasmopara viticola (Berk. \& Curt.) Curt. \& de Toni, é uma doença muito destrutiva, além de ser amplamente distribuída nos plantios dos países produtores de uvas no mundo (24). No Brasil, o míldio ocorre na maioria dos plantios vitícolas (28). No vale do Siriji, no estado de Pernambuco, a ocorrência é acentuada no primeiro semestre do ano, quando as condições ambientais são favoráveis ao desenvolvimento do patógeno, devido aos altos índices pluviométricos. Essa doença afeta todos os órgãos 
verdes da planta. A infecção nos ramos e flores, antes, durante e depois do florescimento, pode resultar na diminuição da quantidade e qualidade dos frutos, e consideráveis perdas de produção podendo atingir até $100 \%$ dependendo das condições de elevada precipitação, alta umidade relativa e longos períodos de umidade $(20,24,28)$.

O controle do míldio realizado pelo uso de fungicidas, no Submédio São Francisco - PE, não tem proporcionado resultados satisfatórios, tendo em vista o grande à agressividade do fungo, que rapidamente alcança níveis significativos de infecção, dificultando seu controle (28, 29). Além disso, o uso inadequado dos produtos sistêmicos pode favorecer o aparecimento de populações resistentes do patógeno, como já foi detectado, desde de 1981, em videiras de alguns países produtores do mundo $(18,24)$. Por outro lado, a redução ou eliminação de agrotóxicos no controle das doenças é um imperativo atual econômico e social. As organizações mundiais como a Organização das Nações Unidas para agricultura e Alimentação FAO e a Organização das Nações Unidas - ONU, buscam alternativas de preservação ambiental, criando normas para gestão e monitoramento ambientais. Assim surgiu a proposta da Produção Integrada de Frutas - PIF para o setor agrícola. Entre as normas que compõem a PIF, está a segurança alimentar que estabelece regras rígidas levando em consideração os resíduos de agroquímicos e a proteção integrada de pragas que estabelece o uso das técnicas preconizadas no manejo integrado de pragas, priorizando o uso de métodos naturais, biológicos e biotecnológicos (5). Considerando esses fatos se faz necessária à utilização de métodos alternativos para o controle dessa doença. Uma alternativa viável pode ser a resistência sistêmica adquirida (RSA) que ativa o sistema de defesa das plantas através de elicitores, bióticos ou abióticos, com a expressão de mecanismos relacionados com a produção de substâncias tóxicas ao patógeno e/ ou formação de barreiras estruturais que restringem a colonização dos tecidos $(7,8,9,16)$. Ativação de defesa natural da planta tem sido demonstrada após a aplicação exógena de compostos como 2,6-dicloroisonicotinico (INA), ácido DL-â-aminobutírico (BABA), acibenzolar-S-methyl (ASM), ácido poliacrílico, tianina $(4,17,19)$, ecolife ${ }^{\circledR}$ (11) e Agro$\operatorname{Mos}^{\circledast}$ (12). O indutor abiótico BABA é um aminoácido que ao ser aplicado sobre a superfície foliar ou no solo, induz a resistência contra vários patógenos foliares e radiculares. O BABA induz a acumulação de PR-proteínas por diferentes caminhos, dependendo do sistema patógeno-hospedeiro (7). O ecolife é composto por bioflavonóides cítricos, ácido ascórbico, fitoalexinas cítricas, ácidos orgânicos e açúcares que bioestimulam as plantas a produzirem suas próprias defesas. Pode ser aplicado alternadamente com outros produtos, é atóxico, não corrosivo e não volátil e tem uso na pré e pós-colheita sem período de carência. Já os produtos, Crop-set ${ }^{\circledR} \mathrm{e}$ Codavit $^{\circledR}$ são fertilizantes compostos por cobre, ferro, manganês e zinco, sendo que o ultimo elemento só encontrado no Codavit. Entre os indutores bióticos, o Agro-Mos que é um mananoligossacarídeo fosforilado derivado da parede da levedura Saccharomyces cerevisae 1026 (Hansen), Improcrop Brasil, Curitiba-PR, e tem demonstrado eficiência no controle de doenças (12). O presente trabalho teve como objetivo verificar a eficácia da aplicação de BABA, Agro-Mos ${ }^{\circledR}+$ Crop-set $^{\circledR}$, Codavit $^{\circledR}$ e Ecolife ${ }^{\circledR}$ na indução de resistência a $P$. viticola, em plantios comerciais de Vitis labrusca L. variedade 'Isabel', visando o controle do míldio, bem como testar modelos linear, monomolecular, logarítmico, exponencial e gomperzt, a fim de verificar qual dos modelos descreveria melhor a severidade do $P$. viticola, durante o período de cultivo da videira, nas condições estudadas.

\section{MATERIAL E MÉTODOS}

\section{Aplicação dos indutores em campos de videira}

Os experimentos foram realizados em três áreas de produção de uvas da variedade 'Isabel', localizadas nos municípios de São Vicente Férrer - PE (área 1), Natuba-PB (área 2) e Macaparana - PE (área 3), no período de março a julho de 2004. Durante o período experimental, registrou-se índice pluviométrico médio de $221 \mathrm{~mm}$, com amplitude de 143-306 mm.

Nas áreas 1 e 2, as aplicações com os indutores foram realizadas quinzenalmente, 20 dias após a brotação das videiras, utilizando-se pulverizador costal manual (Jacto modelo $\mathrm{PJH}$ ) com capacidade de $20 \mathrm{~L}$, pressão variada com a máxima de $6 \mathrm{kgf} / \mathrm{cm}^{3}$, bico de jato cone, perfazendo um total de quatro aplicações, num período de quatro meses, com ácido DL-â-aminobutírico - BABA (50 mg L $\left.{ }^{-1}\right)$; AgroMos - AM + Crop-set - Cs $\left(1 \mathrm{~mL} \mathrm{~L}^{-1} ; 0,3 \mathrm{~mL} \mathrm{~L}^{-1}\right) ; \mathrm{AM}+\mathrm{Cs}(1 \mathrm{~mL}$ $\left.\mathrm{L}^{-1} ; 0,3 \mathrm{~mL} \mathrm{~L}^{-1}\right)$ intercalado com Codavit $-\mathrm{Cd}\left(1 \mathrm{~mL} \mathrm{~L}^{-1}\right)$, Ecolife $-\mathrm{Ec}$ $\left(1,5 \mathrm{~mL} \mathrm{~L}^{-1}\right)$ e testemunha (sem aplicação).

$\mathrm{Na}$ área 3, as aplicações foram realizadas a cada 21 dias. Para a aplicação dos produtos utilizou-se pulverizador costal manual (Jacto modelo PJH) conforme especificações descritas anteriormente, perfazendo um total de três aplicações, com $\mathrm{Cd}\left(1 \mathrm{~mL} \mathrm{~L}^{-1}\right) ; \mathrm{AM}+\mathrm{Cs}$ (1 mL L $\left.\mathrm{mL}^{-1} ; 0,3 \mathrm{~mL} \mathrm{~L}^{-1}\right) ; \mathrm{AM}+\mathrm{Cs}\left(1 \mathrm{~mL} \mathrm{~L}^{-1} ; 0,3 \mathrm{~mL} \mathrm{~L}^{-1}\right)$ intercalado com Cd (1 mL L $\left.\mathrm{mL}^{-1}\right)$, Ec (1,5 mL L $\left.\mathrm{mL}^{-1}\right)$ e testemunha (sem aplicação).

Devido a grave situação em que se encontravam os vinhedos do vale do Siriji com a presença do míldio e o assinalamento da ferrugem, concomitantemente, houve a necessidade de testar outros produtos e combinações dos mesmos, bem como outros períodos de aplicação, a fim de minimizar as perdas produzidas pelas doenças. Nesse sentido foi introduzido no experimento da área 3 , o tratamento com $\mathrm{Cd}$.

\section{Delineamento experimental e avaliação do míldio}

No experimento foi utilizado o delineamento estatístico em blocos ao acaso, constituído de cinco tratamentos, acima citados, e três repetições. Cada área estudada era composta de três blocos. Foram analisadas as variáveis: severidade da doença e Área abaixo da curva de progresso da doença - AACPD [(calculada pela expressão AACPD = $\left\{O ́\left[Y_{i}+Y_{i+1} / 2\right]\left(t_{i+1}-t_{i}\right)\right\}$, onde $Y_{i}$ e $Y_{i+1}$ são valores da severidade observados em duas avaliações consecutivas e $t_{i+1}$ e $t_{i}$ o intervalo entre avaliações (27)]. As avaliações semanais foram efetuadas em 50 folhas da planta central de cada parcela, que era constituída de nove plantas. Em todas as áreas, as amostragens, para avaliação da doença, foram realizadas semanalmente e iniciadas após sete dias da primeira aplicação dos indutores, totalizando sete avaliações.

A severidade foi estimada com o auxílio de escala diagramática, atribuindo-se notas variando de 1 a 6 , descrita por Azevedo (2). As médias da severidade e AACPD foram comparadas pelo teste de Tukey, realizado com o software estatístico Statistical Analysis System (26). Com os dados obtidos ao longo do período experimental, foram testados modelos linear, monomolecular, logístico, exponencial e gompertz.

\section{RESULTADOS E DISCUSSÃO}

Em função dos resultados obtidos, com relação à severidade do míldio da videira, podemos observar que o indutor $\mathrm{AM}+\mathrm{Cs}$ destacouse dos demais tratamentos, entretanto, não diferiu estatisticamente dos indutores $\mathrm{AM}+\mathrm{Cs}$ e $\mathrm{Cd}$, na área 2, e de $\mathrm{AM}+\mathrm{Cs}$ e $\mathrm{Cd}$, e Ec na área 3. Quanto à eficiência de controle observa-se na Tabela 1 que o $\mathrm{AM}+\mathrm{Cs}$ foi capaz de reduzir a severidade da doença em $37,46 \%$, 
Tabela 1. Efeito de indutores abióticos na severidade e na área abaixo da curva de progresso da doença - AACPD de Plasmopara viticola, em três áreas produtoras de uva no estado de Pernambuco

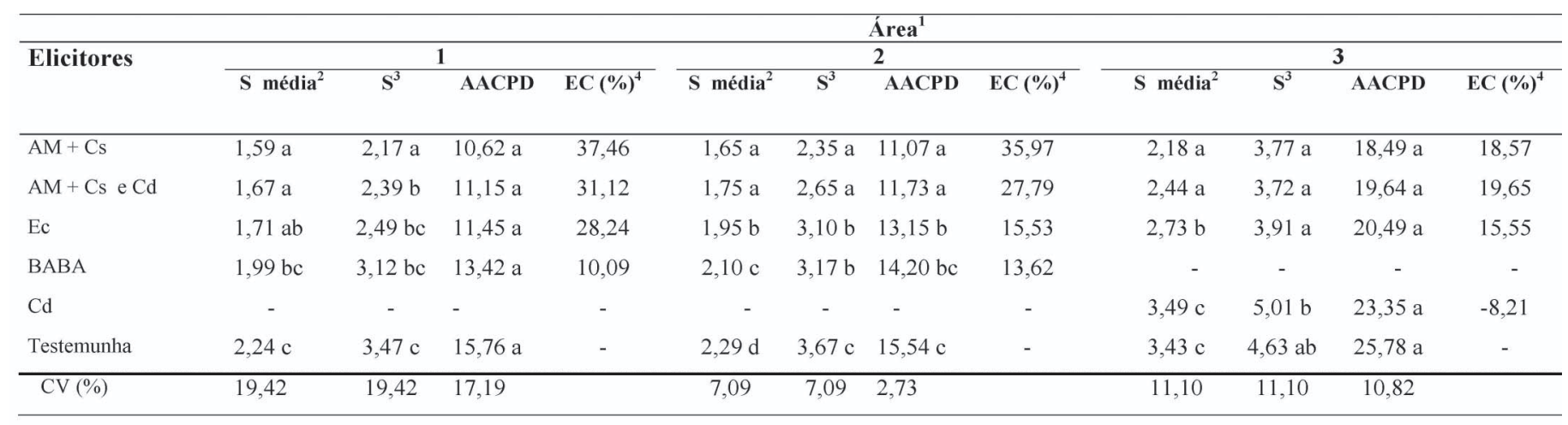

$\mathrm{S}=$ severidade; $\mathrm{EC}=$ Eficiência de controle.

$\mathrm{AM}=$ Agro-Mos; $\mathrm{Cs}=$ Crop-set $; \mathrm{Ec}=$ ecolife; $\mathrm{Cd}=$ codavit; $\mathrm{BABA}=$ ácido DL-â-aminobutírico.

${ }^{1}$ Área 1 = São Vicente Férrer - PE, área 2 = Natuba - PB e área 3 = Macaparana - PE.

${ }^{2}$ Média das três repetições de cada tratamento nas sete avaliações. Em cada coluna média seguida de letras distintas são diferentes entre si, ao nível de $5 \%$ de probabilidade pelo teste de Tukey.

${ }^{3}$ Média de três repetições uma semana após a última aplicação com o indutor. Em cada coluna média, seguida de letras distintas são diferentes entre si, ao nível de $5 \%$ de probabilidade pelo teste de Tukey.

${ }^{4}$ As análises foram realizadas utilizando a fórmula de Abbott (01).

$35,97 \%$ e $18,57 \%$, respectivamente, nas áreas 1,2 e 3 . Comparando o tratamento anterior com $\mathrm{AM}+\mathrm{Cs}$ e Cd este proporcionou menor eficiência de controle nas área 1 e 2, o mesmo não ocorrendo na área 3. $\mathrm{O}$ tratamento com Ec apresentou menor eficiência de controle em relação aos anteriores. Em estudos de doenças pós-colheita os indutores AM e Ec proporcionaram reduções significativas na severidade da antracnose em frutos de mamoeiro (11), manga (12) e maracujazeiro (3). Já os indutores BABA e Cd usados nesse experimento não apresentaram eficiência na indução de resistência para o problema em questão, contrastando nesse sentido com os resultados de campo obtidos por Reuveni et al. (25), na região de Golan em Israel, que utilizaram o indutor BABA ou a mistura deste com fungicidas, em Vitis vinifera L. cvs. 'Cabernet Sauvignon' e 'Chardonnay', com duas aplicações foliares do produto num intervalo de 14 dias e na concentração de $0,1 \%$, suprimindo o desenvolvimento do míldio da videira acima de 90\%. Por outro lado Querino et al. (22) demonstraram que o BABA na dosagem de $2,1 \mathrm{~m} \cdot \mathrm{mL}^{-1}$ e $0,525 \mathrm{mg} \mathrm{mL}^{-1}$ utilizado em banana maçã e Grande Naine, respectivamente, apresentou eficiência de 35,29\% na banana maçã e 21,55\% na Grande Naine, na redução da severidade do Mal do Panamá, em casa de vegetação. Gurgel et al. (14) em estudo de controle da fusariose do tomateiro utilizando os indutores ASM e BABA, em diferentes dosagens e épocas de aplicação concluiu que o BABA, na dosagem $30 \mathrm{mg} \mathrm{mL}^{-1}$, aplicado cinco dias antes da inoculação do patógeno foi o mais eficiente. Contudo, o BABA não se mostrou eficiente quando utilizado no Vale do Siriji, estado de Pernambuco. Vários fatores podem ter contribuído para que esses resultados fossem

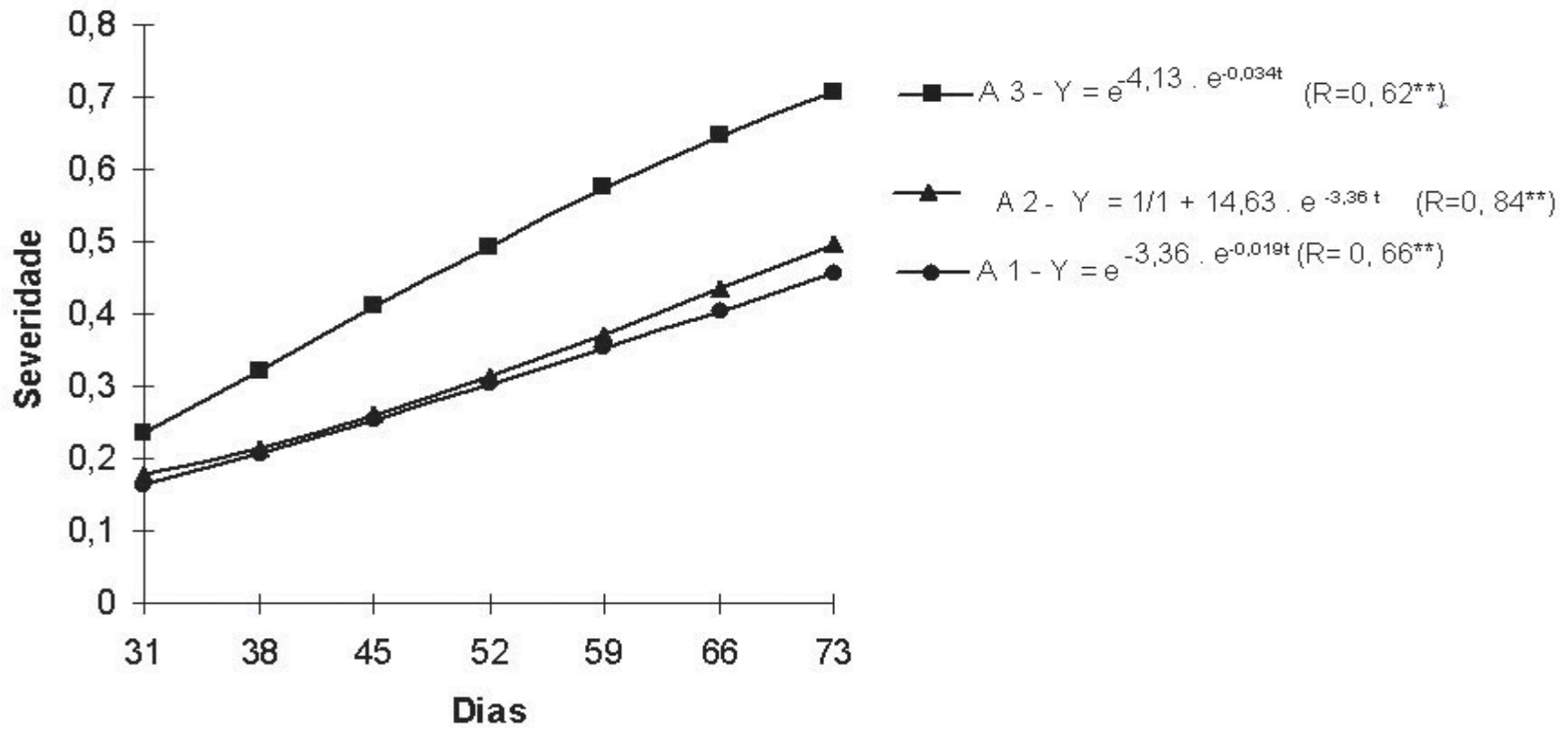

Figura 1. Severidade do míldio da videira, Plasmopara viticola, em três áreas experimentais. A 1 - São Vicente Férrer, A 2 - Natuba e A 3 Macaparana. 


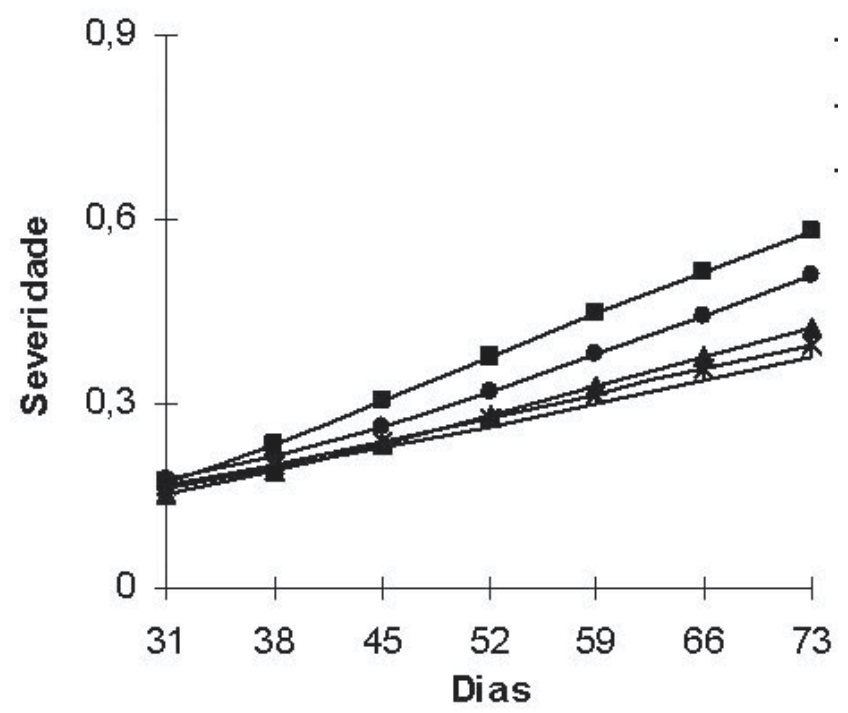

$$
\begin{aligned}
& -A M+C s-Y=e^{-2,85 \cdot e^{-0,015 t}}\left(R=0,76^{\# t}\right) \\
& \rightarrow-A M+C s e C d-Y=e^{-2,88 \cdot e^{-0,0161 t}}\left(R=0,93^{\text {\#t }}\right) \\
& \rightarrow-E C-Y=e^{-3,35 \cdot e^{-0,019+}}\left(R=0,80^{\text {Hit }}\right) \\
& \rightarrow-B A B A-Y=V 1+15,43 \cdot e^{-0,038 t} \quad\left(R=0,69^{* n}\right) \\
& \rightarrow \text {-Testemunha }-Y=e^{-4,2 \cdot e^{-0,028 t}}\left(R=0,86^{\text {\#n }}\right)
\end{aligned}
$$

A

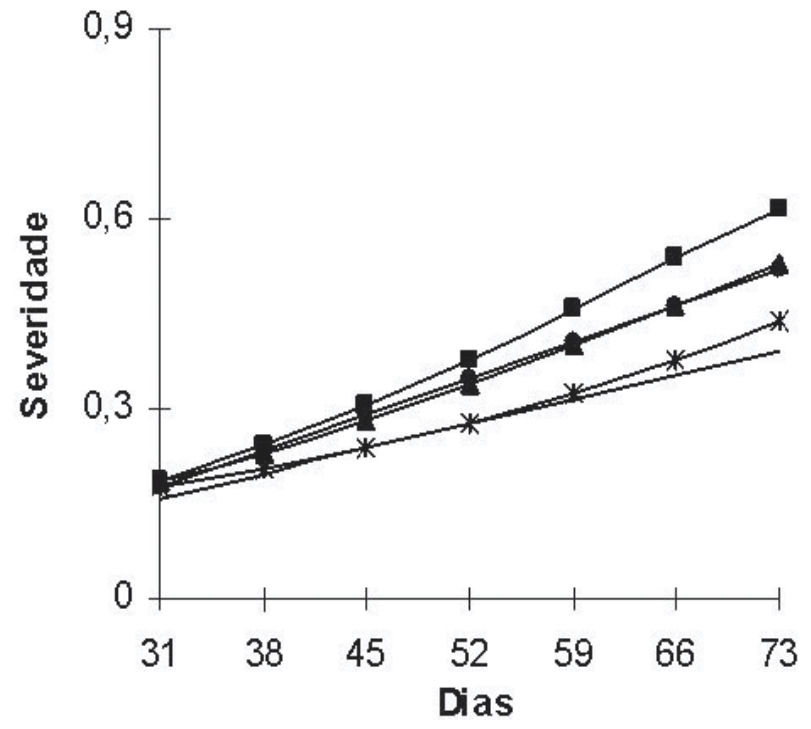

B

$$
\begin{aligned}
& -\mathrm{AM}+\mathrm{Cs}-\mathrm{Y}=-0,012+0,006 \mathrm{t}\left(\mathrm{R}=0,94^{\text {\#N }}\right) \\
& \text { * } \mathrm{AM}+\mathrm{Cs} \text { e Cd }-\mathrm{Y}=0,088 \cdot \mathrm{e}^{\left.-0,022{ }_{(\mathrm{R}} \mathrm{R}=0,91 \mathrm{M}\right)} \\
& \rightarrow-E c-Y=11+14,22 \cdot e^{-0,039}\left(R=0,96^{m}\right)
\end{aligned}
$$$$
\longrightarrow \text { BABA }-Y=-0,077+0,008 \mathrm{t}\left(\mathrm{R}=0,96^{\mathrm{m}}\right)
$$$$
\rightarrow \text {-Testemunha }-Y=11+18,04 \cdot e^{-0,046 t}\left(R=0,96^{*}\right)
$$

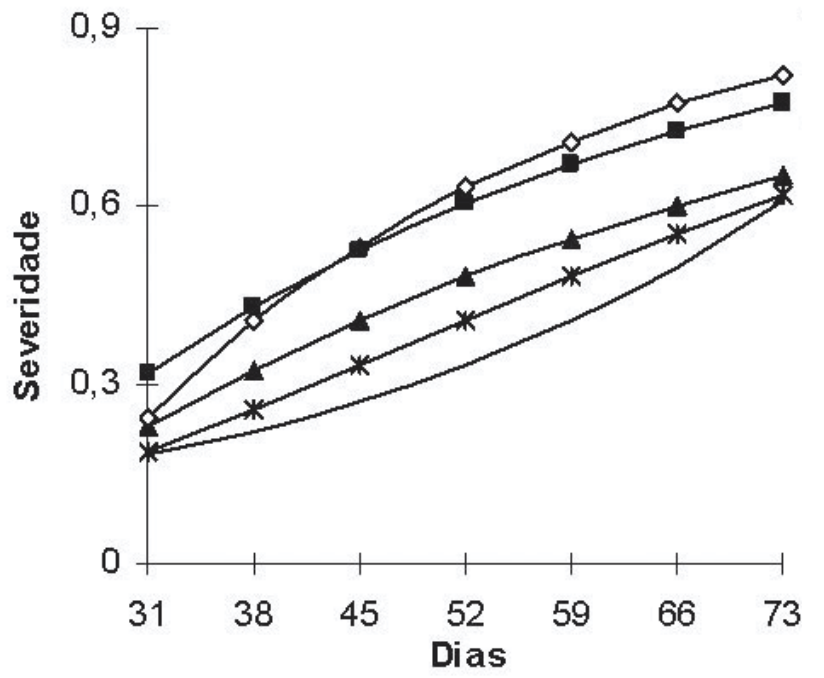

$$
\begin{aligned}
& -A M+C S-Y=0,0743 \cdot e^{-0,029 t}\left(R=0,88^{\text {mat }}\right)
\end{aligned}
$$

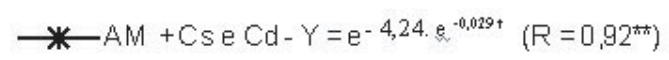

$$
\begin{aligned}
& \text { - } \\
& \leadsto-\mathrm{Cd}-Y=1-2,18 \cdot \mathrm{e}^{-0,034 t}\left(R=0,86^{\mathrm{m}}\right) \\
& \rightarrow-T e s t e m u n h a-Y=1-1,53 \cdot e^{-0,026 t}\left(R=0,84^{\text {\#I }}\right)
\end{aligned}
$$

C

Figura 2. Severidade do míldio da videira, Plasmopara viticola, em plantas tratadas com indutores de resistência, em três plantios comerciais de uva (Vitis labrusca), no período de 73 dias após aplicação dos mesmos. A - São Vicente Férrer, B - Natuba e C - Macaparana. AM = Agro-Mos; Cs = Cropset; $\mathrm{Ec}=$ ecolife; $\mathrm{Cd}=$ codavit; $\mathrm{BABA}=$ ácido DL-â-aminobutírico. 
diferentes, como por exemplo, as dosagens utilizadas, condições edafoclimáticas e a cultivar, que nesse estudo foi a V. labrusca cv. 'Isabel'.

Na Tabela 1 observa-se que nas três áreas estudadas para todos tratamentos utilizados, a severidade do míldio aumentou com o decorrer do tempo. Contudo, na área 3, a severidade foi maior do que as demais, aproximando-se do nível mais alto da escala de notas dessa doença. Um dos fatores que pode ser responsável por esse aumento seria a redução do número (passando de quatro para três) e aumento do intervalo (15 para 21 dias) de aplicações. Outro fator que contribuiu para que não só nessa como em todas as áreas a severidade da doença fosse alta, a ponto de não produzir frutos comercializáveis, foram os índices pluviométricos elevados, observados no período experimental, pois essa doença é especialmente séria em anos com elevada precipitação, alta umidade relativa e longos períodos de umidade sobre as folhas e frutos, com perdas que podem atingir $100 \%$ da produção de um vinhedo (10).

Em todas as áreas, para a maioria dos tratamentos, não ocorreu uma correlação entre as duas variáveis avaliadas, severidade e AACPD, excetuando-se o tratamento, da área 3, onde se aplicou o indutor $\mathrm{Cd}$, que foi significativa ao nível de $5 \%$ de probabilidade pelo teste $\mathrm{t}(\mathrm{R}=$ 0,99*), indicando que existe uma correlação positiva entre as duas variáveis, ou seja, quanto maior a severidade maior será a AACPD.

Um dos aspectos mais importantes na análise temporal de epidemias é a seleção de um modelo apropriado para descrever à curva de progresso da doença. Esta seleção tem como objetivo estimar parâmetros que são utilizados na análise estatística para a comparação entre curvas de progresso de doenças (6). Os modelos matemáticos testados no ajuste das curvas de progresso do míldio da videira (linear, monomolecular, logístico, exponencial e gompertz) mostraram que nas áreas 1, 2 e 3 a severidade da doença desenvolveu-se obedecendo as seguintes equações, gompertz $\mathrm{Y}=\mathrm{e}^{-3,36 \cdot \mathrm{e}^{-0,019 \mathrm{t}}}\left(\mathrm{R}=0,66^{* *}\right)$, logístico

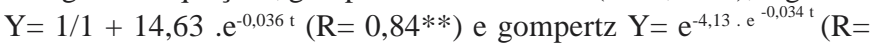
$0,62 * *)$, respectivamente (Figura 1). A severidade do míldio da videira em função do tempo, na área 1 , na maioria dos tratamentos obedeceram ao modelo de gompertz, exceto o tratamento com BABA cujo modelo que se ajustou foi o logístico (Figura $2 \mathrm{~A}$ ) concordando com os dados da severidade média e eficiência de controle relacionado na Tabela 1, onde o tratamento com o indutor BABA mostrou-se menos eficiente e com a severidade média alta, não diferindo da testemunha. Para as parcelas tratadas com $\mathrm{AM}+\mathrm{Cs}$ a severidade foi expressa pela equação $\mathrm{Y}=\mathrm{e}^{-2,85 \cdot \mathrm{e}^{-0,015 \mathrm{t}}}\left(\mathrm{R}=0,76^{* *}\right)$. Na área 2, o aumento da severidade da doença nos tratamentos estudados não apresentou uma predominância de um modelo, mesmo assim a eficiência de controle foi acima de $25 \%$ nos tratamentos $\mathrm{AM}+\mathrm{Cs}$ e $\mathrm{AM}+\mathrm{Cs}$ e $\mathrm{Cd}$ que obedeceram aos modelos linear $\mathrm{Y}=-0,012+0,006 \mathrm{t}(\mathrm{R}=0,94 * *)$ e exponencial $\mathrm{Y}=$ $0,088 . \mathrm{e}^{-0,022 \mathrm{t}}(\mathrm{R}=0,91 * *)$, respectivamente (Figura $2 \mathrm{~B}$ ). O modelo monomolecular apareceu com uma freqüência maior do que os demais, nos tratamentos da área 3. Para os tratamentos AM + Cs, AM + Cs e $\mathrm{Cd}$, e $\mathrm{E}$ os modelos que melhor descreveram a severidade da doença foram exponencial $\mathrm{Y}=0,0743 . \mathrm{e}^{-0,029 \mathrm{t}}\left(\mathrm{R}=0,88^{* *}\right)$, gombertz $\mathrm{Y}=\mathrm{e}^{-}$ 4,24 . e $-0,028 \mathrm{t}(\mathrm{R}=0,92 * *)$ e monomolecular $\mathrm{Y}=1-1,39 \cdot \mathrm{e}^{-0,019} \mathrm{t}(\mathrm{R}=$ $0,65^{* *}$ ), respectivamente (Figura $2 \mathrm{C}$ ), no entanto a maior eficiência de controle foi do tratamento AM + Cs e Cd concordando com o modelo geral apresentado para a severidade da área 3 (Figura 1). Esses modelos podem ser utilizados em sistemas de previsão ou aviso para Míldio da videira aumentando conseqüentemente a eficiência de controle do mesmo, tendo em vista que modelos simplificados de um ou mais componentes do ciclo da doença (germinação, infecção, esporulação, etc.) podem ser usados para predizer o risco relativo do desenvolvimento da mesma $(13,15)$. De um modo geral, o manejo do
Míldio, na maioria das áreas de produção, geralmente requer o uso de fungicidas aplicado varias vezes durante o ciclo da cultura. Visando diminuir os custos das aplicações de fungicidas e o desejo de reduzir o nível desses produtos no meio ambiente consideráveis esforços têm sido realizados para desenvolver sistemas de previsão ou aviso para essa doença $(13,21,23)$.

Em função dos resultados obtidos e nas condições em que foi realizado o trabalho, sugeri-se que o uso do indutor de resistência Agro-Mos + Crop-set é uma das ferramentas que pode ser utilizada no manejo do controle da doença, para reduzir o número de aplicações de fungicidas no meio ambiente e, conseqüentemente, o impacto ambiental. Com relação aos modelos obtidos se faz necessário a realização de mais estudos, visando obter maiores informações de parâmetros relativos aos fatores ambientais e ciclo da doença, para Vitis labrusca L. variedade 'Isabel'.

\section{AGRADECIMENTOS}

Aos produtores Manuel Vicente da Silva, Miguel Borba e Humberto Coelho por cederem as áreas para instalação do experimento e apoio logístico na execução do mesmo. Os autores também agradecem o apoio da prefeitura Municipal de São Vicente Férrer pelo auxilio financeiro dado à pesquisa.

\section{REFERÊNCIAS BIBLIOGRÁFICAS}

1.Abbott, W.S. A method of computing the effectiveness of an insecticide. Journal Economic Entomology, Lanham, v.8, p. 265-267, 1925 2.Azevedo, L.A.S. Manual de quantificação de doenças de plantas. São Paulo: Luiz Antonio Siqueira de Azevedo, 1997. 114p.

3.Benato, E.A.; Sigrist, J.M.M.; Hanashiro, M. M.; Magalhães, M.J.M.A.; Binotti, C.S. Avaliação de fungicidas e produtos alternativos no controle de podridões pós-colheita em maracujá amarelo. Summa Phytopathologica, Botucatu, v. 28, n.4, p.299-304, 2002.

4.Benhamou, N.; Kloepper, J.W.; Tuzun, S. Induction of resistance against Fusarium wilt of tomato by combination of chitosan with an endophytic bacterial strain: ultra structure and cytochemistry of the host response. Planta, Heidelberg, v.204, p.153-168, 1998.

5.Brasil. Instrução Normativa n. ${ }^{\circ}$ 20, de 27 de setembro de 2001. Aprova as Diretrizes Gerais para a Produção Integrada de Frutas - DGPIF e as Normas Técnicas Gerais para a Produção Integrada de Frutas - NTGPIF. Diário Oficial [da] União, Brasília, Df, 15, out. 2001.

6.Campbell, C.L.; Madden, L.V. Introduction to plant disease epidemiology. New York. Wiley-Interscience. 1990.

7.Cohen, Y. The BABA story of induced resistance. Phytoparasitica, Bet Dagan, v.29, n.5, p.1-4, 2001.

8.Cohen, Y. â-aminobutyric acid-induced resistance against plant pathogens. Plant Disease, St. Paul, v.86, n.5, p. 448-457, 2002.

9.Cohen,Y., Reuveni, M.; Baider, A. Local and systemic activity of BABA (DL-3-aminobutyric acid) against Plasmopara viticola in grapevines. European Journal of Plant Pathology, Dordrecht, v.105, p.351$361,1999$.

10.Czermainski, A. B. C.; Sônego, O. R. Influencia das condições climáticas sobre a eficácia de fungicidas empregados para o controle do míldio em Vitis vinifera. Ciência Rural, Santa Maria, v. 34, n. 1, p. 5-11, 2004.

11.Dantas, S.A.F.; Oliveira, S.M.A.; Bezerra Neto, E.; Coelho, R.S.B.; Silva, R.L.X. Indutores de resistência na proteção do mamão contra podridão pós-colheita. Summa Phytopathologica, Botucatu, v. 30, n.3, p. 77-87, 2002.

12.Dantas, S.A.F.; Tavares, S.C.C. de H.; Oliveira, S.M.A.; Coelho, R.S.B.; Cavalcanti, V.A.L B.; Silva, R.L.X. Indutores de resistência a patógenos Pós-colheita de manga. Summa Phytopathologica, Botucatu, v. 30, p. 314-319, 2004.

13.Ellis, M.A.; Madden, L.V.; Lalancette, N. A diseasse forecastimg program for grape downy mildew in Ohio. In: Gadoury, D.M.; Seem, R.C. 
(Ed.). Proc. Int. Worshop Grapevine Downy Mildew Modeling. N.Y.: Agric. Exp. Stn. Sep. Rep, 1994. p.92-95.

14.Gurgel, L.M.S.; Oliveira, S.M.A.; Coelho, R.S.B.; Silva, R.L.X. Proteção a murcha de fusário do tomateiro com Acibenzolar-S-Metil e Ácido â-aminobutirico, em campo. Fitopatologia Brasileira, Fortaleza, v.30, n.6, p.655-657. 2005.

15.Kasseenmeyer, H.H. Experience with electronic warning of Downy Mildew of grapevine In: Gadoury, D.M.; Seem, R.C. (Ed.). Proc. Int. Worshop Grapevine Downy Mildew Modeling. N.Y.: Agric. Exp. Stn. Sep. Rep, 1994. p.80-81.

16.Kuc, J. Concepts and direction of induced systemic resistance in plants and its application. European Journal of Plant Pathology, Dordrecht, v.107, p.7-12, 2001.

17.Laun, N. Induces resistance: first experiences with Bion in vegetables. Gemüse, Munchen, v.34, n.5, p.294-297, 1997.

18.Leroux, P.; Clerjeau, M. Resistance of Botrytis cinerea and Plasmopara viticola to fungicides in the French vineyards. Crop Protection, West Sussex, v.4, p.137-160, 1985.

19.Lyon, G.D.; Reglinski, T.; Forrest, R.S.; Newton, A.C. The use of resistance elicitors to control plant diseases. Aspects of Applied Biology, v.42, p.227-234, 1995.

20.Madden, L.V, Ellis, M.A., Lalancette, N., Hughes, G., Wilson, L.L. Evaluation of disease warning system for downy mildew of grapes. Plant Disease, St. Paul, v. 84, n. 25, p.549-554, 2000.

21.Magery, P.; Wachtel, M.F.; Weir, P.C.; Seem, R.C. A computer-based Simulator for rational management of grapevine downy mildew (Plasmopara viticola). In: Gadoury, D.M.; Seem, R.C. (Ed.). Proc.
Int. Worshop Grapevine Downy Mildew Modeling. N.Y.: Agric. Exp. Stn. Sep. Rep, 1994. p.82-91.

22.Querino, C. M. B.; Laranjeira, D.; Coelho, R. S. B.; Matos, A. P. Efeito de dois indutores de resistência sobre a severidade do maldo-Panamá. Fitopatologia Brasileira, Fortaleza, v.30, n.3, p.239-243, 2005.

23.Park, E.W.; Seem, R.C.; Gadoury, D.M.; Pearson, R.C. DMCAST: A predicton model for grape downy mildew development. Vitic. Enol. Sci., Davis, v.52, p. 182-189, 1997.

24.Pearson, R.C.; Goheen, A.C. Compendium of Grape Disease. St. Paul: APS Press, 1988. 93 p.

25.Reuveni, M., Zahavi, T.; Cohen, Y. Controlling Downy Mildew (Plasmopara viticola) in field-grown grapevine with $\beta$-aminobutyric acid (BABA). Phytoparasitica, Rehovot, v.29, n.2, p. 1-9, 2001.

26.SAS Institute. JMP statistics and graphics guide, Version 3.1. Cary, 1995

27.Shaner, G.; Finney, R.E. The effect of nitrogen fertilization on the expression of slow-mildewing resistance in knox wheat. Phytopathology, St. Paul, v.67, n.8, p.1051-1056, 1977.

28.Tavares, S.C.C. de H.; Lima, M.F.; Melo, N.F. Principais doenças da videira e alternativas de controle. In: Sousa Leão P.C.; Soares J.M. A viticultura no semi-árido brasileiro. Petrolina: Embrapa SemiÁrido, 2000. v 1., cap. 12, p. 293-346.

29.Tavares, S.C.C. de H.; Cruz, S.C. Doenças causadas por fungos. In: Lima, M.F.; Moreira, W.A. Uva de mesa fitossanidade. Brasília: EMBRAPA informação tecnológica, 2002. v.1, cap.1, p.9-26. 